\title{
Efficacy of time pressure management in stroke patients with slowed information processing: a randomized controlled trial
}

Citation for published version (APA):

Winkens, I., van Heugten, C. M., Wade, D. T., Habets, E., \& Fasotti, L. (2009). Efficacy of time pressure management in stroke patients with slowed information processing: a randomized controlled trial. Archives of Physical Medicine and Rehabilitation, 90(10), 1672-1679. https://doi.org/10.1016/j.apmr.2009.04.016

Document status and date:

Published: 01/01/2009

DOI:

10.1016/j.apmr.2009.04.016

Document Version:

Publisher's PDF, also known as Version of record

Document license:

Taverne

Please check the document version of this publication:

- A submitted manuscript is the version of the article upon submission and before peer-review. There can be important differences between the submitted version and the official published version of record.

People interested in the research are advised to contact the author for the final version of the publication, or visit the DOI to the publisher's website.

- The final author version and the galley proof are versions of the publication after peer review.

- The final published version features the final layout of the paper including the volume, issue and page numbers.

Link to publication

\footnotetext{
General rights rights.

- You may freely distribute the URL identifying the publication in the public portal. please follow below link for the End User Agreement:

www.umlib.nl/taverne-license

Take down policy

If you believe that this document breaches copyright please contact us at:

repository@maastrichtuniversity.nl

providing details and we will investigate your claim.
}

Copyright and moral rights for the publications made accessible in the public portal are retained by the authors and/or other copyright owners and it is a condition of accessing publications that users recognise and abide by the legal requirements associated with these

- Users may download and print one copy of any publication from the public portal for the purpose of private study or research.

- You may not further distribute the material or use it for any profit-making activity or commercial gain

If the publication is distributed under the terms of Article 25fa of the Dutch Copyright Act, indicated by the "Taverne" license above, 


\title{
Efficacy of Time Pressure Management in Stroke Patients With Slowed Information Processing: A Randomized Controlled Trial
}

\author{
Ieke Winkens, PhD, Caroline M. Van Heugten, PhD, Derick T. Wade, MD, Esther J. Habets, \\ Luciano Fasotti, PhD
}

ABSTRACT. Winkens I, Van Heugten CM, Wade DT, Habets EJ, Fasotti L. Efficacy of Time Pressure Management in stroke patients with slowed information processing: a randomized controlled trial. Arch Phys Med Rehabil 2009;90:1672-9.

Objective: To examine the effects of a Time Pressure Management (TPM) strategy taught to stroke patients with mental slowness, compared with the effects of care as usual.

Design: Randomized controlled trial with outcome assessments conducted at baseline, at the end of treatment (at $5-10 \mathrm{wk}$ ), and at 3 months.

Setting: Eight Dutch rehabilitation centers.

Participants: Stroke patients $(\mathrm{N}=37$; mean age $\pm \mathrm{SD}$, $51.5 \pm 9.7 \mathrm{y}$ ) in rehabilitation programs who had a mean Barthel score \pm SD at baseline of $19.6 \pm 1.1$.

Intervention: Ten hours of treatment teaching patients a TPM strategy to compensate for mental slowness in real-life tasks.

Main Outcome Measures: Mental Slowness Observation Test and Mental Slowness Questionnaire.

Results: Patients were randomly assigned to the experimental treatment $(n=20)$ and to care as usual $(n=17)$. After 10 hours of treatment, both groups showed a significant decline in number of complaints on the Mental Slowness Questionnaire. This decline was still present at 3 months. At 3 months, the Mental Slowness Observation Test revealed significantly higher increases in speed of performance of the TPM group in comparison with the care-as-usual group $(t=-2.7, P=.01)$.

Conclusions: Although the TPM group and the care-asusual group both showed fewer complaints after a 3-month follow-up period, only the TPM group showed improved speed of performance on everyday tasks. Use of TPM treatment therefore is recommended when treating stroke patients with mental slowness.

Key Words: Cognitive therapy; Information processing; Rehabilitation; Stroke.

From Vilans, Hoensbroek, (Winkens, Habets); the School for Mental Health and Neurosciences (Winkens, Van Heugten) and the Care and Public Health Research Institute (Wade), Maastricht University, Maastricht; the Centre of Excellence in Rehabilitation De Hoogstraat, Rudolf Magnus Institute, Utrecht (Van Heugten), the Rehabilitation Foundation Limburg, Hoensbroek (Wade); Sint Maartenskliniek Research, Development \& Education, Nijmegen (Fasotti); Nijmegen Institute for Cognition and Information, Nijmegen (Fasotti), The Netherlands; the Oxford Centre for Enablement, Oxford, United Kingdom (Wade).

Supported partly by the Dutch Brain Foundation (grant no. 13F05[2].16).

No commercial party having a direct financial interest in the results of the research supporting this article has or will confer a benefit on the authors or on any organization with which the authors are associated.

Correspondence to Ieke Winkens, PhD, Vilans, PO Box 192, 6430 AD Hoensbroek, The Netherlands, e-mail: i.winkens@vilans.nl. Reprints are not available from the author.

0003-9993/09/9010-00936\$36.00/0

doi:10.1016/j.apmr.2009.04.016
(C) 2009 by the American Congress of Rehabilitation Medicine

$\mathbf{M}$ ENTAL SLOWNESS IS a common complaint after stroke and traumatic brain injury. ${ }^{1,2}$ Patients may present with a wide range of problems and complaints. They may manifest an externally observed slowness, as evidenced by their slow performance on neuropsychologic tasks, and they may have problems with everyday situations in which events proceed at a rate they cannot control. Conversations, driving a car, and watching television are daily life tasks in which these problems may become apparent. Also, patients may subjectively feel that things happen too quickly, that they can no longer keep up mentally with the cognitive demands being made by external events. These problems, in turn, may lead to feelings of fatigue, altered mood, and irritability. ${ }^{3,4}$ The frequency of these complaints and their impact on the lives of stroke patients make mental slowness a prime target for cognitive rehabilitation.

One seemingly obvious rehabilitation method for cognitive deficits is the so-called direct stimulation approach, in which remediation interventions are aimed at specifically impaired cognitive processes. For mental slowness this means that patients are trained to perform reaction time tasks at a normal rate. The underlying ideas are that the target process can be improved by repetitive stimulation and that this improvement generalizes to several related skills and to daily life. Both ideas appear to be questionable given the many remedial programs based on this approach and the modest results obtained, especially with regard to generalization. ${ }^{5-7}$

Fasotti et $\mathrm{al}^{8}$ used a different approach towards mental slowness, namely TPM. TPM consists of 2 types of cognitive strategies by which subjects learn either to "prevent" or to "manage" time pressure, and hence compensate for their slowness and deal with the task at hand, instead of trying to restore a normal speed of information processing. The idea is that TPM treatment is applicable to a broad range of problems caused by mental slowness and hence might generalize more easily to nontrained tasks.

The rationale of TPM can be illustrated by Michon's task analysis, ${ }^{9}$ which was originally designed to describe traffic behavior in hierarchically ordered levels. For any task, decision making can be hierarchically ordered into 3 levels, differing in the amount of time pressure present. The first level, the "strategic level," concerns decisions (and subsequent actions) that can be undertaken well beforehand. When driving a car, for

List of Abbreviations

ADLs activities of daily living

TPM Time Pressure Management 
instance, decisions are made with regard to the route and time of departure. For these decisions there usually is enough time, and no time pressure is experienced. The second level is the "tactical level," which relates to anticipating events and adapting behavior before time pressure builds up. To stay with the example of car driving, the choice of driving speed and car following distance might be adaptations at the tactical level. At this level, time pressure is present but usually still manageable. The third level is the "operational level," where immediate decisions and actions are required to prevent failure and risks. For example, hitting the brakes and turning the wheel to avoid a collision, or reacting to sudden deviations and maneuvering the car through traffic are all operational actions. Here much time pressure is felt.

The basic idea is that the strategic and tactical decision levels are barely affected by mental slowness, and one can greatly reduce time pressure at the operational level by using the preserved ability to make strategic and tactical decisions. In other words, TPM treatment focuses on the reorganization of the execution of actions with time pressure. By breaking up such actions into subtasks and subsequently identifying the amount of time pressure in these subtasks, one can pinpoint decisions and actions that can prevent (strategic) or manage (tactical) time pressure. Patients are taught to move as many actions as possible from the impaired levels with high time pressure to the preserved levels with little or no time pressure. This should minimize the negative consequences of mental slowness and should lead to improvements in task performance. Returning to the driving example for instance, patients may be taught to make strategic decisions, such as leaving early, or tactical decisions, such as keeping an ample distance and not driving too fast. This should give the patient enough time to react in tricky traffic circumstances and even prevent the occurrence of dangerous situations in the first place.

Fasotti et $\mathrm{al}^{8}$ evaluated TPM treatment in a randomized controlled trial. Twenty-two patients with severe to very severe closed head injury were randomly assigned to TPM treatment or concentration training in which verbal instruction was the key element. The results indicated that TPM treatment produced greater gains than concentration training for an information intake task and also appeared to generalize to other measures of speed and memory function. However, this study was small with a limited number of patients, and to date, there are no other studies and no data on the treatment effects of this strategy training for stroke patients.

The goal of the present study was to determine in a randomized controlled trial the efficacy of TPM treatment for stroke patients with mental slowness. We hypothesize that after TPM treatment, patients with stroke will perform better on everyday tasks and will have fewer complaints than patients receiving "care as usual."

\section{METHODS}

Patients who had sustained a stroke at least 3 months earlier and had been referred for cognitive rehabilitation for mental slowness (both inpatients and outpatients) were included in the present study. Referral occurred on the basis of the usual clinical diagnosis of the respective treatment team (including neuropsychologic examinations and clinical interviews). Exclusion criteria were (1) age younger than 18 years; (2) stroke occurred less than 3 months ago; (3) very severe or disabling premorbid or current (continuing) pathologic conditions, such as psychiatric diseases (eg, depression) or substance abuse for which the patient had been hospitalized, or other medical or neurologic diseases (such as tumor or whiplash); or (4) such severe cognitive, communication, physical, or psychologic problems that the patient was unable to perform the tasks, based on the clinical judgment of the treating team.

All patients received verbal and written information about the study and gave written consent to participate. The study protocol was approved by the ethics committee of all participating institutions.

\section{Study Design}

A multicenter, randomized, single-blind, controlled trial design was used to compare the effect of TPM with the effect of care as usual. For each of the 8 participating rehabilitation centers, a random assignment list was created before the start of the trial. After selection and recruitment, an independent person used the list to assign the patients to the TPM treatment group or care as usual group.

Trainers were asked to give all patients 10 hours of their allocated treatment and to record the exact amount of treatment hours. Treatment was given in sessions of $1,1.5$, or 2 hours a week (depending on the learning potential of the patient and usual practice in the particular rehabilitation center).

Baseline measurements were done immediately after inclusion. After the end of the 10-hour treatment period, a second measurement took place, and the final measurement was a follow-up 3 months after the end of the treatment. Inpatients were contacted via their treating psychologist, whereas outpatients were contacted directly at home. Measurements were done by a research assistant (E.J.H.) who was unaware of the allocation of patients. Success of blinding was checked afterwards.

At the start of the study, every participating center decided which trainer was responsible for the TPM treatment. This trainer received a 3-hour course in TPM. Other trainers were responsible for the care as usual.

Time Pressure Management. TPM treatment was given in 3 main stages. ${ }^{10,11}$ The initial aim is to enhance the patient's awareness that mental slowness is a critical problem, that many activities are adversely affected by the inability to react to external events with adequate speed, and that mental slowness may bring about secondary problems such as emotional changes, fatigue, or forgetfulness.

The second stage focuses on the acceptance and acquisition of the TPM strategy. Patients are told that the speed of information processing is not expected to return to premorbid levels, but that there is a strategy called "let me give myself enough time" that might help deal with their slowness. The essence of this 4-step strategy is shown in appendix 1.

The last stage of TPM treatment focuses on generalization. Patients are taught that therapists cannot possibly treat all the problems and tasks patients will encounter in their own surroundings. Therefore, transfer of skills and strategies from the rehabilitation setting to the home setting and from trained to nontrained tasks and situations is of great importance. In this stage, various tasks and situations are trained under more distracting and difficult conditions (see Winkens et $\mathrm{al}^{11}$ for a detailed description).

There are 2 classes of TPM strategies: (1) "preventive strategies" in which the patient learns to anticipate time pressure as much as possible by making decisions while enough time is still available (optimization of task preparation and reduction of stress during task execution due to uncertainty); and (2) "management strategies" in which the patient learns to prevent the increase of time pressure or to deal with the existing level of time pressure during execution of the task. Patients in the TPM group learn to use both kinds of strategies while performing an information intake task (see "Measurements"). When the patient is able to perform the information intake task while 
using the strategy, role plays and real-life situations are practiced.

Care as usual. The other group received care as usual. Because 8 different centers participated in the study, content of the care as usual varied. Most centers restricted their cognitive training for slowed information processing to giving education about brain damage, and speed of information processing and its possible consequences for daily functioning, and gave practical hints and advice about how to deal with these consequences. One center gave actual practice training more similar to TPM, training patients in performing tasks while using compensation strategies.

The essential difference between the 2 treatment methods is that TPM is directly aimed at behavioral, cognitive change in treatment situations. The training starts with psychoeducation on mental slowness and time pressure, and patients are clearly told that they will receive treatment to diminish time pressure. Then, during treatment, patients are constantly monitored and trainers are directly involved in the teaching of new strategies. Feedback is frequently given, and possible errors are corrected on the spot. In the care as usual group, the focus is not on diminishing time pressure. In addition, patients are not specifically trained in performing everyday tasks. So, TPM is not only more detailed and task specific, but also more directly aimed at improving task execution, whereas the control treatment assumes that an intermediate stage, in which the patient is supposed to transform instructions into actions, should take place spontaneously.

\section{Measurements}

Six primary and 13 secondary outcome measures were assessed by an independent, blinded research assistant.

Information intake task. ${ }^{8}$ In this task, patients are told they are about to buy a waterbed and are required to ask for more information about this item. The shop assistant's answers are shown on a video. The instructions emphasize that the patients should remember as much information as possible, and they are allowed to do anything to make this easier. At the end, the patient's reproduction score is calculated by adding up the reproduced items. The maximum score is 21 .

Mental Slowness Observation Test. ${ }^{4}$ This test consists of 4 tasks that are commonplace for most people, are brief, and amenable to accurate timing. The tasks are specifically designed to measure performance in time pressure situations. Patients are required to handle incoming information and to work within certain time limits. The tasks are (1) following a route description, (2) sorting money, (3) making a telephone call, and (4) looking up telephone numbers. Two tasks have a preset maximum time: subtask 2 (sorting money, $5 \mathrm{~min}$ ) and subtask 4 (looking up telephone numbers, 10min). If the subject does not complete the task within this period, the item is terminated. Otherwise the tester records the time needed to perform each task (Time score) and the number of elements correctly achieved (Elements score). The maximum score per task is 10 , and the maximum total score is 40 .

For both the Mental Slowness Observation Test and the information intake task, the number of strategies used is counted (eg, interrupting the video, reiterating the information) and used as outcome measures.

Mental Slowness Questionnaire. ${ }^{4}$ This questionnaire consists of 21 items examining different kinds of daily activities that are likely to be related to mental slowness. Examples of the items are, "I have trouble following a conversation," or "I have trouble doing 2 things at the same time." Each item is scored on a 5-point frequency scale ranging from 0 (this never happens) to 4 (this happens often). Each problem is also scored on a 3-point severity scale ranging from 0 (not troublesome) to 2 (very troublesome). The score on the frequency scale is multiplied by the score on the severity scale. For this weighted scale the maximum total score is 168 .

In addition to the primary outcome measures described, measures for personal ADLs, fatigue, depression, and quality of life, and neuropsychologic tests for speed of information processing, memory, attention, and executive functioning also were administered.

Independence in personal ADLs was measured using the Barthel Index. ${ }^{12}$ The researcher filled in the questionnaire on the basis of the patient's reports. The maximum score is 20 . A higher score means higher independence.

Fatigue was measured using the Fatigue Severity Scale, ${ }^{13}$ a self-report instrument used to assess levels of fatigue and its effect on daily functioning. It contains 9 items, each of which is scored on a 7-point scale. Then a mean score is calculated. Patients with higher scores are more fatigued.

Mood was measured using the Center for Epidemiologic Studies Depression Scale, ${ }^{14}$ a self-report questionnaire screening for depression in stroke patients. It contains 20 items, each of which is scored on a 4-point scale ranging from less than 1 day a week to 5 to 7 days a week. A higher score means more depressive complaints.

Quality of life was measured using the EuroQol-5D, ${ }^{15}$ which consists of 2 parts, the self-classifier or questionnaire, and the EQ-VAS or Thermometer. The EQ-5D self-classifier is a 1-page questionnaire that captures respondents' descriptions of health problems on a 5-dimensional classification of mobility, self-care, usual activities, pain and discomfort, and anxiety and depression. Each dimension is rated by respondents on a 3-level scale from 1 (no problem) to 3 (unable or extreme problem). The EQ-VAS is a $20-\mathrm{cm}$ visual analog scale, portrayed as similar to a thermometer, on which respondents rate their health state today between 0 (worst imaginable) to 100 (best imaginable).

Speed of information processing was measured using the Symbol Digit Modalities Test. Patients are instructed to transcribe from symbols to numerals, in writing and verbally, using a key matching particular symbols to particular numerals. Subjects are required to complete as much of the task as possible within 90 seconds. ${ }^{16}$ The number of correct responses is evaluated.

Speed of information processing was also measured using the Paced Auditory Serial Addition Task. ${ }^{17}$ The task was presented at a pacing with a 3.2-second interstimulus interval. The number of correct responses is evaluated. simple reaction time was measured on a pocket personal computer. Subjects touch the screen of the computer until a stimulus appears, then release the screen as fast as possible. Reaction times are measured.

Memory was measured using the Auditory Verbal Learning Test, ${ }^{18}$ which measures episodic memory. The total number of correct responses is calculated.

Attention was measured using the Trail Making Test parts A and B. ${ }^{19}$ Time and error rate are measured.

Executive functioning was measured using the Stroop Color Word Task. $^{20}$ The variable of interest is the time needed to complete each subtest.

\section{Statistical Analysis}

Descriptive statistics were used to present patient characteristics and test results at baseline. Between-group differences at baseline were investigated by chi-square analysis, Fisher exact test, or independent samples $t$ test as appropriate. 


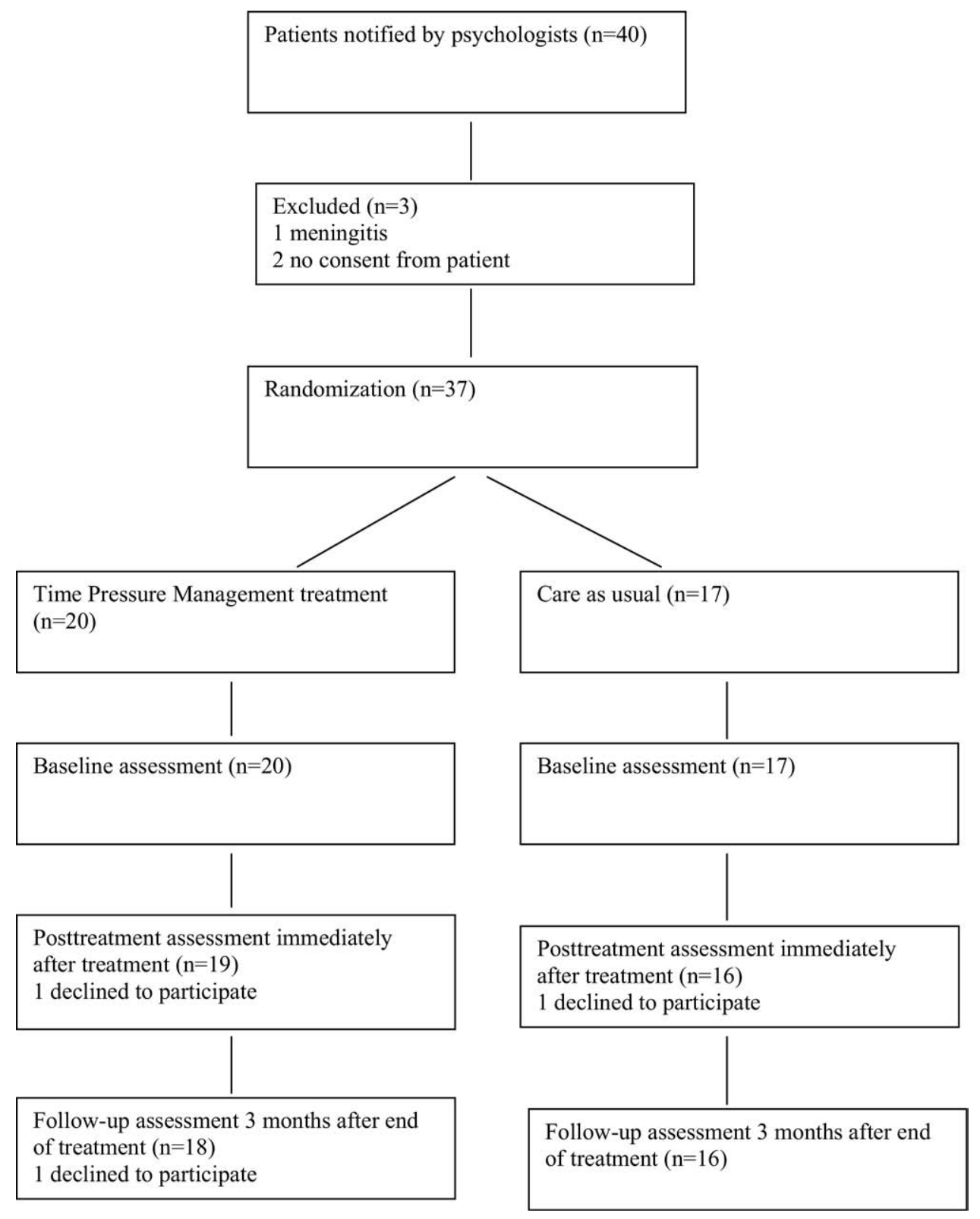

Fig 1. Flow chart of patients in the trial.

Intervention effects were examined with linear regression analyses. For the variables that turned out to be skewed, nonparametric Mann-Whitney $U$ tests were used. To evaluate outcome, change scores and $95 \%$ confidence intervals were calculated by subtracting baseline scores from posttreatment and follow-up scores, respectively (to improve precision in controlling for influence of baseline score on outcome). Between-group differences in change scores were investigated for all outcome measures. To further improve the power of the linear regression analyses, outcome at baseline was included as a covariate in the analyses. A 2-sided significance test (significance level, .05) was used because we did not have an assumption on the direction of these differences in advance. Analyses were carried out using SPSS for Windows, version 15.0. ${ }^{\mathrm{a}}$

\section{RESULTS}

Figure 1 shows the patient flow and follow-up to 3 months after the end of the treatment. Patients were recruited from November 2005 until October 2007. During the study, 40 patients with a reduced speed of information processing were referred for treatment in the participating institutions; 37 were included.

Of the 20 patients allocated to TPM treatment, 1 declined participation before the start of the treatment, 3 patients stopped treatment after 1 hour because of insufficient motivation, and 2 patients stopped after 7 hours of TPM treatment because both the patient and the trainer agreed that no further gains were to be achieved. Fourteen patients received exactly 10 hours of training as indicated by the TPM protocol. 
Table 1: Baseline Characteristics for Patients Included in the Study

\begin{tabular}{|c|c|c|}
\hline Demographics & $\operatorname{TPM}(n=20)$ & Care as Usual $(n=17)$ \\
\hline Age $(y)$ & $49.5 \pm 8.0$ & $53.9 \pm 11.1$ \\
\hline \multicolumn{3}{|l|}{ Sex, n (\%) } \\
\hline Men & $9(45)$ & $12(71)$ \\
\hline Women & $11(55)$ & $5(29)$ \\
\hline \multicolumn{3}{|l|}{ Education, n (\%) } \\
\hline Low & $6(30)$ & $3(18)$ \\
\hline Middle & $10(50)$ & $8(47)$ \\
\hline High & $4(20)$ & $6(35)$ \\
\hline Time since stroke (mo) & $19.3 \pm 29.6$ & $6.9 \pm 5.4$ \\
\hline Barthel score & $19.7 \pm 1.3$ & $19.6 \pm 0.9$ \\
\hline \multirow[t]{2}{*}{ Allocated treatment $(\mathrm{h})$} & Total group $(n=19): 8.1 \pm 3.2$ & $9.3 \pm 3.0$ \\
\hline & $\begin{array}{l}\text { Group receiving training according to } \\
\text { protocol }(n=14): 9.5 \pm 1.6\end{array}$ & \\
\hline Additional treatment (h) & $21.1 \pm 17.3(n=19)$ & $30.5 \pm 15.7$ \\
\hline \multicolumn{3}{|l|}{ Primary outcome measures } \\
\hline Information intake task (no. of used strategies) & $0.5 \pm 0.5$ & $0.5 \pm 0.6$ \\
\hline Information intake task (reproduction score) & $10.6 \pm 4.1$ & $9.6 \pm 4.2$ \\
\hline MSOT (no. of used strategies) & $4.6 \pm 2.1$ & $3.8 \pm 1.6$ \\
\hline MSOT (no. of correct elements) & $34.2 \pm 8.0$ & $32.3 \pm 7.1$ \\
\hline MSOT (time in seconds) & $861.0 \pm 255.0$ & $871.4 \pm 172.3$ \\
\hline MSO (frequency $\times$ severity score) & $44.6 \pm 41.1$ & $62.3 \pm 43.8$ \\
\hline \multicolumn{3}{|l|}{ Secondary outcome measures } \\
\hline FSS score & $30.0 \pm 16.3$ & $32.7 \pm 10.7$ \\
\hline CES-D score & $9.0 \pm 7.7$ & $13.6 \pm 6.9$ \\
\hline EuroQOL VAS score & $70.5 \pm 19.2$ & $65.9 \pm 17.4$ \\
\hline \multicolumn{3}{|l|}{ Neuropsychologic tests } \\
\hline SDMT_writing (no. correct) & $36.0 \pm 11.6$ & $31.3 \pm 10.8$ \\
\hline SDMT_reading (no. correct) & $45.9 \pm 12.4$ & $39.4 \pm 11.7$ \\
\hline Simple reaction time test (time in seconds) & $0.31 \pm 0.10$ & $0.34 \pm 0.06(n=16)$ \\
\hline PASAT (no. correct), & $38.2 \pm 11.7(n=19)$ & $32.4 \pm 15.1(n=16)$ \\
\hline AVLT (no. correct) & $52.9 \pm 9.5(n=19)$ & $46.1 \pm 10.4(n=15)$ \\
\hline TMT A (time in seconds) & $44.5 \pm 20.9$ & $46.2 \pm 16.0$ \\
\hline TMT B (time in seconds) & $90.7 \pm 38.4(n=19)$ & $131.3 \pm 102.5$ \\
\hline Stroop Color Word Test card 1 (time in seconds) & $52.6 \pm 11.9(n=19)$ & $62.9 \pm 26.3$ \\
\hline Stroop Color Word Test card 2 (time in seconds) & $67.0 \pm 14.7(n=19)$ & $87.5 \pm 51.9$ \\
\hline Stroop Color Word Test card 3 (time in seconds) & $105.6 \pm 23.4(n=19)$ & $119.7 \pm 36.9(n=15)$ \\
\hline
\end{tabular}

NOTE. Values are mean \pm SD unless otherwise noted.

Abbreviations: AVLT, Auditory Verbal Learning Test; CES-D, Center for Epidemiologic Studies Depression Scale; FSS, Fatigue Severity Scale; MSOT, Mental Slowness Observation Test; MSQ, Mental Slowness Questionnaire; PASAT, Paced Auditory Serial Addition Task; QOL, quality of life; SDMT, Symbol Digit Modalities Test; TMT, Trail Making Test; VAS, visual analog scale.

\section{Comparability}

Patient characteristics and baseline results are displayed in table 1. The results show that patients in both groups were relatively young for a stroke population and physically independent. In addition, table 1 shows that patients in both groups were not severely depressed or excessively tired.

At baseline, the participants in both groups did not differ significantly on any of the observed variables (see table 1). Although not statistically significant, we considered the difference in time since stroke between the 2 groups sufficient clinically to include time since stroke as a covariate in the analyses.

The amount of treatment that patients received did not differ significantly between the 2 intervention groups $(t=1.2$, $P>.05)$. Patients in the care as usual group had a mean \pm SD of $9.3 \pm 5.4$ hours of treatment. Patients in the TPM group had $8.1 \pm 3.2$ hours of TPM treatment. However, when restricting the TPM group to the 14 patients who did not deviate from the TPM protocol, the TPM group had $9.5 \pm 1.6$ hours of TPM treatment, which also did not differ significantly from the care as usual group $(t=-.03, P>.05)$. Furthermore, the 2 intervention groups did not differ with respect to the total amount of hours spent in additional treatments, such as occupational therapy, physiotherapy, psychoeducation, recreational therapy, and social work.

The effectiveness of blinding of the research assistant (E.J.H.) who performed the outcome measurements was checked post hoc. Analyses showed that she had guessed the allocation correctly in 24 of 37 cases (Cohen $\kappa=.29, P>.05$ ).

\section{Posttreatment Outcome}

Table 2 shows the results immediately at the end of the treatment. The 2 groups differed significantly in the use of strategies on the trained information intake task. The TPM group showed an average improvement of 0.4 strategies, while the care as usual group showed a decline of 0.3 strategies $(z=-2.1, P<.05)$. On all other variables (primary as well as secondary outcome measures and neuropsychologic tests), no significant between-group differences were found. 
Table 2: Treatment Effects at the End of Treatment for Both the Experimental and Care as Usual Group

\begin{tabular}{|c|c|c|c|c|c|c|c|}
\hline \multirow[b]{2}{*}{ Primary Outcome Measures } & \multicolumn{3}{|c|}{ TPM $(n=19)$} & \multicolumn{3}{|c|}{ Care as Usual $(n=16)$} & \multirow[b]{2}{*}{$P$} \\
\hline & $\begin{array}{l}\text { Posttreatment } \\
\text { (Mean } \pm \text { SD) }\end{array}$ & $\begin{array}{l}\text { Mean Change } \\
\text { From Baseline }\end{array}$ & $95 \% \mathrm{Cl}$ & $\begin{array}{l}\text { Posttreatment } \\
\text { (Mean } \pm \text { SD) }\end{array}$ & $\begin{array}{l}\text { Mean Change } \\
\text { From Baseline }\end{array}$ & $95 \% \mathrm{Cl}$ & \\
\hline $\begin{array}{l}\text { Information intake task (no. of } \\
\text { used strategies) }\end{array}$ & $0.8 \pm 1.0$ & 0.4 & -0.1 to 0.8 & $0.3 \pm 0.4$ & -0.3 & -0.7 to 0.1 & .03 \\
\hline $\begin{array}{l}\text { Information intake task } \\
\text { (reproduction score) }\end{array}$ & $11.0 \pm 5.1$ & 0.4 & -1.3 to 2.1 & $9.5 \pm 2.9$ & -0.3 & -2.1 to 1.6 & .60 \\
\hline MSOT (no. of used strategies) & $8.9 \pm 5.1$ & 4.4 & 2.3 to 6.6 & $7.6 \pm 3.8$ & 3.8 & 2.1 to 5.5 & .60 \\
\hline MSOT (no. of correct elements) & $34.1 \pm 5.9$ & -0.1 & -2.2 to 2.1 & $33.7 \pm 6.9$ & 1.2 & -2.1 to 4.6 & .50 \\
\hline MSOT (time in seconds) & $796.9 \pm 246.9$ & -73.4 & -142.0 to -4.7 & $848.1 \pm 205.0$ & -18.9 & -73.7 to 35.8 & .20 \\
\hline MSQ (frequency $\times$ severity score) & $36.6 \pm 37.7$ & -9.6 & -22.8 to 3.6 & $49.1 \pm 42.2$ & -10.3 & -26.7 to 6.1 & .70 \\
\hline
\end{tabular}

NOTE. Linear regression analyses adjusted for baseline scores on the specific outcome measures and time since stroke, or nonparametric Mann-Whitney $U$ tests are used; a 2-sided significance test is used.

Abbreviations: $\mathrm{Cl}$, confidence interval; MSOT, Mental Slowness Observation Test; MSQ, Mental Slowness Questionnaire.

Both groups showed an improvement in the mean number of strategies used on the Mental Slowness Observation Test, with the TPM group showing a mean increase of 4.4 strategies after treatment (98\% improvement), and the care as usual group showing a mean increase of 3.8 strategies after treatment (100\% improvement). Both groups also showed a considerable decline in complaints on the Mental Slowness Questionnaire, with the TPM group showing an average decline of 9.6 points ( $21 \%$ decline), and the care as usual group showing an average decline of 10.3 points (17\% decline). In addition, the TPM group showed an average decline of 73.4 seconds needed to complete the Mental Slowness Observation Test compared with an average decline of 18.9 seconds in the care as usual group (9\% difference in the TPM group compared with $2 \%$ difference in the care as usual group). However, the differences in change scores between the 2 groups were not statistically significant.

\section{Outcome at Follow-Up}

Table 3 shows the follow-up versus baseline results for both intervention groups. At 3 months, the 2 groups differed significantly on the time needed to complete the Mental Slowness Observation Test. The TPM group showed an average decline of 154.6 seconds in the time needed to complete the test, compared with an average decline of 26.5 seconds in the care as usual group (18\% improvement in the TPM group vs only $3 \%$ improvement in the care as usual group); this was statistically significant $(t=-2.7, P=.01)$. After controlling for time since stroke, the larger decrease in the time needed to complete the test in the TPM group was still significant $(t=$ $-2.9, P<.01$ ). On all other variables (primary as well as secondary outcome measures and neuropsychologic tests), no significant between-group differences were found.

At the 3-month follow-up, the number of strategies used in both the information intake task and the Mental Slowness Observation Test had returned to baseline levels. However, the TPM group showed an average decline of 10.5 points on the number of complaints on the Mental Slowness Questionnaire (22\% decline), and the care as usual group showed an average decline of 10.3 points ( $28 \%$ decline). Between-group differences, however, were not statistically significant.

A per protocol analysis was conducted, excluding all patients in the TPM group who were not treated according to the study protocol $(n=5)$. Results of the per protocol analysis were similar to the results of the intention-to-treat analysis.

\section{DISCUSSION}

In this trial, evidence was found for the long-term efficacy of TPM treatment in stroke patients with mental slowness, suggesting that strategy training benefits take some time to become evident. Immediately after treatment, the patients in the experimental group showed a clinically significant decline in the number of complaints on the Mental Slowness Questionnaire and a major decrease in the time needed to complete the Mental Slowness Observation Test. However, the results of the experimental group were not significantly

Table 3: Treatment Effects at 3 Months for Both the Experimental and Care as Usual Group

\begin{tabular}{|c|c|c|c|c|c|c|c|}
\hline \multirow[b]{2}{*}{ Primary Outcome Measures } & \multicolumn{3}{|c|}{ TPM $(n=17)$} & \multicolumn{3}{|c|}{ Care as Usual $(n=16)$} & \multirow[b]{2}{*}{$P$} \\
\hline & $\begin{array}{l}\text { Follow-Up } \\
\text { (Mean } \pm \mathrm{SD} \text { ) }\end{array}$ & $\begin{array}{l}\text { Mean Change } \\
\text { From Baseline } \\
\text { to Follow-Up }\end{array}$ & $95 \% \mathrm{Cl}$ & $\begin{array}{l}\text { Follow-Up } \\
\text { (Mean } \pm \text { SD) }\end{array}$ & $\begin{array}{l}\text { Mean Change } \\
\text { From Baseline } \\
\text { to Follow-Up }\end{array}$ & $95 \% \mathrm{Cl}$ & \\
\hline $\begin{array}{l}\text { Information intake task (no. of } \\
\text { used strategies) }\end{array}$ & $0.6 \pm 0.6$ & 0.1 & -0.2 to 0.5 & $0.5 \pm 0.5$ & -0.1 & -0.4 to 0.3 & .40 \\
\hline $\begin{array}{l}\text { Information intake task } \\
\text { (reproduction score) }\end{array}$ & $10.9 \pm 5.0$ & 0.6 & -1.1 to 2.3 & $11.1 \pm 4.6$ & 1.3 & -0.6 to 3.2 & .50 \\
\hline MSOT (no. of used strategies) & $4.9 \pm 2.6$ & 0.5 & -1.0 to 1.9 & $4.9 \pm 1.8$ & $1.0(n=15)$ & 0.3 to 1.7 & .90 \\
\hline MSOT (no. of correct elements) & $34.9 \pm 6.3$ & 0.5 & -2.0 to 3.1 & $34.0 \pm 5.5$ & $1.6(n=15)$ & -0.9 to 4.0 & .70 \\
\hline MSOT (time in seconds) & $715.6 \pm 247.2$ & -154.6 & -212.4 to -96.7 & $840.1 \pm 288.0$ & $-26.5(n=15)$ & -112.4 to 59.4 & .01 \\
\hline MSQ (frequency $\times$ severity score) & $38.6 \pm 47.7$ & -10.5 & -27.2 to 6.3 & $42.9 \pm 44.8$ & -16.6 & -26.0 to -7.1 & .20 \\
\hline
\end{tabular}

NOTE. Linear regression analyses adjusted for baseline scores on the specific outcome measures and time since stroke, or nonparametric Mann-Whitney $U$ tests are used; a 2-sided significance test is used.

Abbreviations: $\mathrm{Cl}$, confidence interval; MSOT, Mental Slowness Observation Test; MSO, Mental Slowness Questionnaire. 
different statistically from the results of the control group. At 3 months, however, the experimental group showed a statistically significant decrease in the time needed to complete the Mental Slowness Observation Test, compared with the care as usual group. Patients in the TPM group were more efficient in completing everyday tasks such as following a route description or making a telephone call; they needed less time without becoming less accurate.

This pattern of improvement in the experimental group, starting during treatment but continuing (and becoming statistically significant) thereafter, is likely to occur in a strategy training model, where one might expect some benefit as the strategy is implemented, but increasing benefit as it becomes increasingly automated and integrated into a wider range of behaviors. ${ }^{21}$ Patients need time to accept and learn that the strategy is helpful and probably also will be in other situations than the ones that are trained.

For this study, "dose" of treatment was fixed at a maximum of 10 hours. Some patients may need less ( 2 patients finished after 7 hours). It is unlikely that every patient needs the same amount of treatment to benefit. Some probably need more time to learn to use cognitive strategies such as the ones taught in TPM treatment. In several other studies, patients receive treatment for at least 15 hours. ${ }^{22-24}$ Given the small but significant effect in the everyday tasks, it is reasonable to predict that an expanded version of treatment would produce greater effects, at least in some patients. Extending TPM treatment over more sessions would give trainers the opportunity to incorporate more practice situations into the treatment, thereby increasing the chance for generalization to other nontrained tasks and situations to occur.

The results support previous findings from the study by Fasotti et al, ${ }^{8}$ the only other study on the effects of TPM. In that study, 12 patients with slowed speed of information processing after traumatic brain injury received TPM treatment, and 10 other patients received regular concentration training. The patients in the TPM group improved considerably in the use of strategies and remembered more information in an information intake task compared with the control group. In addition, the results in our study are consistent with positive results of other studies on different kinds of strategy training (eg, for neglect, apraxia, memory impairment, attention deficits). ${ }^{25}$

\section{Study Limitations}

The following comments can be made concerning our study. First, although the TPM treatment seems to be effective, the small study sample makes conclusions uncertain. Although 8 centers participated in the study, recruitment was poor. Other impairments possibly but not necessarily related to slowness, such as fatigue, memory problems, or language problems, might have had treatment priority.

Second, both intervention groups were selected groups consisting of relatively young, ADL-independent people. As such, we have to be careful in generalizing the results found in this study to other groups of stroke patients - that is, older and ADL-dependent stroke patients. However, our experiences with TPM treatment have given us no reason to assume that it is not possible to apply this treatment in different settings and to other patient groups. We do think that success of treatment is dependent on patients' awareness of and the severity of their cognitive deficits. ${ }^{11}$

In this study, a patient's level of awareness of deficits was not an exclusion criterion. However, patients with high awareness and motivation are most likely to benefit, whereas patients with severely compromised awareness and denial of their prob- lems are least likely to benefit. ${ }^{11,26}$ Although not measured objectively in this study, some trainers spontaneously reported that some patients were not able to appreciate their deficits and the benefits of using the strategy. A larger effect of TPM treatment possibly would have been found if patients had been selected on their level of awareness.

\section{CONCLUSIONS}

Although both our study and the only other study ${ }^{8}$ on the effects of TPM treatment were small, results of the 2 studies are promising. First, there are indications for positive longterm effects of the strategies taught in TPM treatment for patients with traumatic brain injury as well as for stroke patients. Second, our multicenter study shows that the treatment can be taught to other therapists. We think that this intervention can be implemented easily into the structure of care as it is currently delivered, and can in the future be included as a part of usual rehabilitation care for inpatients as well as outpatients.

More studies are needed to confirm the effects of TPM treatment, and we recommend conducting new studies that include a higher number of less selected patients. In addition, it would be worthwhile to use longer treatment and follow-up periods. The results in this study indicate that it takes a while for strategies to become incorporated into patients' everyday lives. It would also be interesting to see whether effects are still apparent after, for example, a year, or whether additional treatment is needed to prevent patients from forgetting what they have learned during treatment.

\section{APPENDIX 1: THE TPM STRATEGY "LET ME GIVE MYSELF ENOUGH TIME TO DO THE TASK"}

\begin{tabular}{|c|c|}
\hline Questions to be Asked & Main Objective \\
\hline $\begin{array}{l}\text { 1. Are there } 2 \text { or more things to } \\
\text { be done at the same time? } \\
\text { Could I be overwhelmed or } \\
\text { distracted? }\end{array}$ & $\begin{array}{l}\text { To recognize time pressure } \\
\text { in the task at hand. }\end{array}$ \\
\hline $\begin{array}{l}\text { 2. Which things can be done } \\
\text { before the actual task } \\
\text { begins? Make a plan. }\end{array}$ & $\begin{array}{l}\text { To prevent as much time } \\
\text { pressure as possible. }\end{array}$ \\
\hline $\begin{array}{l}\text { 3. What to do in case of } \\
\text { unexpected, overwhelming } \\
\text { time pressure? Make an } \\
\text { emergency plan. }\end{array}$ & $\begin{array}{l}\text { To deal with time pressure } \\
\text { as quickly and effectively } \\
\text { as possible. }\end{array}$ \\
\hline $\begin{array}{l}\text { 4. Plan and emergency plan } \\
\text { ready? Then use it regularly. }\end{array}$ & $\begin{array}{l}\text { Urging the patient to } \\
\text { monitor himself while } \\
\text { using the TPM strategy. }\end{array}$ \\
\hline
\end{tabular}

Data from Fasotti et al. ${ }^{8}$

\section{References}

1. Hochstenbach J. The cognitive, emotional, and behavioural consequences of stroke [dissertation]. Nijmegen: Catholic University Nijmegen; 1999

2. Visser-Keizer AC, Meyboom-de Jong B, Deelman BG, Berg IJ, Gerritsen MJ. Subjective changes in emotion, cognition and behaviour after stroke: factors affecting the perception of patients and partners. J Clin Exp Neuropsychol 2002;24:1032-45.

3. Winkens I, Van Heugten CM, Fasotti L, Duits AA, Wade DT. Manifestations of mental slowness in the daily life of stroke patients. Clin Rehabil 2006;20:827-34.

4. Winkens I, Van Heugten CM, Fasotti L, Wade DT. Reliability and validity of two new instruments for measuring aspects of mental slowness in the daily lives of stroke patients. Neuropsychol Rehabil 2009;19:64-85. 
5. Robertson HI. Does computerised cognitive rehabilitation work? A review. Aphasiology 1990;4:381-405.

6. Wilson BA. Assessment and management of memory problems. In: Von Steinbuechel N, Von Cramon DY, Poeppel E, editors. Neuropsychological rehabilitation. Berlin: Springer Verlag; 1992. p 194-202.

7. Halligan PW, Wade DT. The effectiveness of rehabilitation for cognitive deficits. Oxford: Oxford Univ Pr; 2005.

8. Fasotti L, Kovacs F, Eling PA, Brouwer WH. Time Pressure Management as a compensatory strategy training after closed head injury. Neuropsychol Rehabil 2000;10:47-65.

9. Michon JA. Dealing with danger. Summary report of a workshop in the Traffic Research Centre. Groningen: State University Groningen, The Netherlands; 1979.

10. Ylvisaker M, Szekeres SF, Henry K, Sullivan DM, Wheeler P. Topics in cognitive rehabilitation theory. In: Ylvisaker M, Gobble EM, editors. Community re-entry for head injured adults. Boston: College-Hill Pr; 1987. p 137-220.

11. Winkens I, Van Heugten C, Wade D, Fasotti L. Training patients in Time Pressure Management, a cognitive strategy for mental slowness. Clin Rehabil 2009;23:79-90.

12. Collin C, Wade DT, Davies S, Home V. The Barthel ADL Index: a reliability study. Int Disabil Stud 1988;10:61-3.

13. Krupp LB, LaRocca NG, Muir-Nash J, Steinberg AD. The Fatigue Severity Scale: application to patients with multiple sclerosis and systemic lupus erythematosus. Arch Neurol 1989;46:1121-3.

14. Shinar D, Gross CR, Price TR, Banko M, Bolduc PL, Robinson RG. Screening for depression in stroke patients: the reliability and validity of the Center for Epidemiologic Studies Depression Scale. Stroke 1986;17:241-5.

15. Brooks R. EuroQOL: the current state of play. Health Policy 1996;37:53-72.

16. Smith A. Symbol Digit Substitution Test (SDMT). Manual (revised). Los Angeles: Western Psychological Services; 1982.
17. Gronwall DM, Sampson H. The psychological effects of concussion. Auckland: Auckland Univ Pr; 1974.

18. Brand PA, Jolles J. Learning and retrieval rate of words presented auditory and visually. J Gen Psychol 1985;112:201-10.

19. Reitan RM. Validity of the Trail Making Test as an indicator of organic brain damage. Percept Mot Skills 1958;8:271-6.

20. Houx PJ, Jolles J, Vreeling FW. Stroop interference: aging effects assessed with the Stroop Color-Word Test. Exp Aging Res 1993; 19:204-24

21. Gray JM, Robertson I, Pentland B, Anderson S. Microcomputer-based attentional retraining after brain damage: a randomized group controlled trial. Neuropsychol Rehabil 1992;2:97115 .

22. Donkervoort M, Dekker J, Stehmann-Saris FC, Deelman BG. Efficacy of strategy training in left hemisphere stroke patients with apraxia: a randomized controlled trial. Neuropsychol Rehabil 2001;11:549-66.

23. Kaschel R, Della Sala S, Cantagallo A, Fahlbock A, Laaksonen $\mathrm{R}$, Kazen M. Imagery mnemonics for the rehabilitation of memory: a randomized controlled trial. Neuropsychol Rehabil 2002;12:127-53

24. Pizzamiglio L, Antonucci G, Judica A, Montenero P, Razzano C, Zoccolotti P. Cognitive rehabilitation of the hemineglect disorder in chronic patients with unilateral right brain damage. J Clin Exp Neuropsychol 1992;14:901-23.

25. Cicerone KD, Dahlberg C, Malec JF, et al. Evidence-based cognitive rehabilitation: updated review of the literature from 1998 through 2002. Arch Phys Med Rehabil 2005;86:1681-92.

26. Levine B, Robertson IH, Clare L, et al. Rehabilitation of executive functioning: an experimental-clinical validation of Goal Management Training. J Int Neuropsychol Soc 2000;6:299-312.

\section{Supplier}

a. SPSS Inc, 233 S Wacker Dr, 11th Fl, Chicago, IL 60606. 